The most widely read and highly cited peer-reviewed neurology journal The Official Journal of the American Academy of Neurology

\title{
Spinal Cord Injury, Vertebral Artery Dissection, and Cerebellar Strokes After Chiropractic Manipulation
}

\section{Author(s):}

Gabriel Bortoli Ramos, MD ${ }^{1}$; Rebecca Ranzani Martins, MD ${ }^{1}$; Julia Carvalhinho Carlos de Souza, MD ${ }^{1}$; Fernando Cavalcanti de Sá e Benevides Falcão, MD ${ }^{1}$; Cesar Castello Branco Lopes, MD ${ }^{1}$; Andre Luiz Gargioni de Andrade, MD ${ }^{1}$; Guilherme Diogo Silva, MD ${ }^{1}$

Corresponding Author:

Gabriel Bortoli Ramos gabrielbortoli@icloud.com

Neurology® Published Ahead of Print articles have been peer reviewed and accepted for publication. This manuscript will be published in its final form after copyediting, page composition, and review of proofs. Errors that could affect the content may be corrected during these processes. 
Affiliation Information for All Authors: 1.Hospital das Clinicas da Faculdade de Medicina da Universidade de São Paulo

Contributions:

Gabriel Bortoli Ramos: Major role in the acquisition of data; Study concept or design

Rebecca Ranzani Martins: Drafting/revision of the manuscript for content, including medical writing for content

Julia Carvalhinho Carlos de Souza: Drafting/revision of the manuscript for content, including medical writing for content

Fernando Cavalcanti de Sá e Benevides Falcão: Drafting/revision of the manuscript for content, including medical writing for content

Cesar Castello Branco Lopes: Drafting/revision of the manuscript for content, including medical writing for content

Andre Luiz Gargioni de Andrade: Major role in the acquisition of data

Guilherme Diogo Silva: Drafting/revision of the manuscript for content, including medical writing for content

Number of characters in title: 103

Abstract Word count:

Word count of main text: 100

References: 2

Figures: 1

Tables: 0

Neuroimage Legend Count: 50

Supplemental: Patient consent-to-disclose forms

Search Terms: [ 6 ] Infarction, [ 119 ] CT, [ 120 ] MRI, [ 255 ] Spinal cord trauma; see Trauma/spinal cord trauma (S), [ 266 ] Spinal cord trauma

Study Funding: The authors report no targeted funding

Disclosures: The authors report no disclosures relevant to the manuscript. 
A 48-year-old woman with chronic neck pain presented with a history of sudden neck pain and generalized weakness during a chiropraxis session. Neurological examination showed tetraplegia with C5 sensory level. Cervical spine CT revealed a fracture affecting $\mathrm{C} 5$ and $\mathrm{C} 6$ vertebra (Figure A-B). Cervical spine MRI confirmed spinal cord injury (Figure C). Also, bilateral vertebral artery occlusion and acute cerebellar infarction were found (Figures D-F). Bony ankylosis was found in cervical CT spine suggesting undiagnosed ankylosing spondylitis was a risk factor for spine fracture. Other neurological lesions related to chiropraxis include vertebral artery dissection, epidural hematoma and acute disk hernition ${ }^{1,2}$.

\section{Appendix 1. Authors}

\begin{tabular}{lll} 
Name & Location & Contribution \\
\hline Mabriel Bortoli, & Hospital das & Designed and \\
& Clínicas da & conceptualized the study; \\
& Faculdade de & drafted the manuscript for \\
& Medicina da & intellectual content \\
& Universidade de & \\
& São Paulo São & \\
& Paulo, Brazil & \\
\hline Rebeca Martins, & Hospital das & Data curation \\
MD & Clínicas da & \\
& Faculdade de & \\
& Medicina da & \\
& Universidade de & \\
& São Paulo São & \\
& Paulo, Brazil & \\
Hospital das & Data curation \\
Clínicas da & \\
Faculdade de & \\
Medicina da & \\
Universidade de & \\
& São Paulo São & \\
&
\end{tabular}




\section{Paulo, Brazil}

\begin{tabular}{lll}
\hline Fernando & Hospital das & Data Curation \\
Benevides, MD & Clínicas da & \\
& Faculdade de & \\
& Medicina da & \\
& Universidade de & \\
& São Paulo São & \\
& Paulo, Brazil & \\
\hline Cesar Lopes, MD & Hospital das & Revised the manuscript for \\
& Clínicas da & intellectual content \\
& Faculdade de & \\
& Medicina da & \\
& Universidade de & \\
& São Paulo São & \\
Paulo, Brazil & \\
\hline Guilherme D. & Hospital das & Revised the manuscript for \\
Silva, MD & Clínicas da & intellectual content \\
& Faculdade de & \\
& Medicina da & \\
& Universidade de & \\
& São Paulo São & \\
& Paulo, Brazil & \\
& & \\
& & \\
\hline
\end{tabular}

1. Nielsen, Sabrina Mai, et al. "The risk associated with spinal manipulation: an overview of reviews." Systematic reviews 6.1 (2017): 1-19.

2. Johnson, lan. "Adverse effects of spinal manipulation." Journal of the Royal Society of Medicine 100.10 (2007): 444-445. 
Figure. Spinal cord injury, vertebral artery dissection, and cerebellar strokes after chiropractic manipulation

Sagittal cervical spine CT revealed a fracture and intense bony ankylosis (Figures $A$ and B). T2-weighted sagittal cervical spinal cord MRI showed a spinal cord injury (Figure C). CT angiography identified bilateral vertebral artery occlusion (Figure D). Axial diffusionweighted imaging and FLAIR brain MRI documented cerebellar ischemia (Figure E, F).

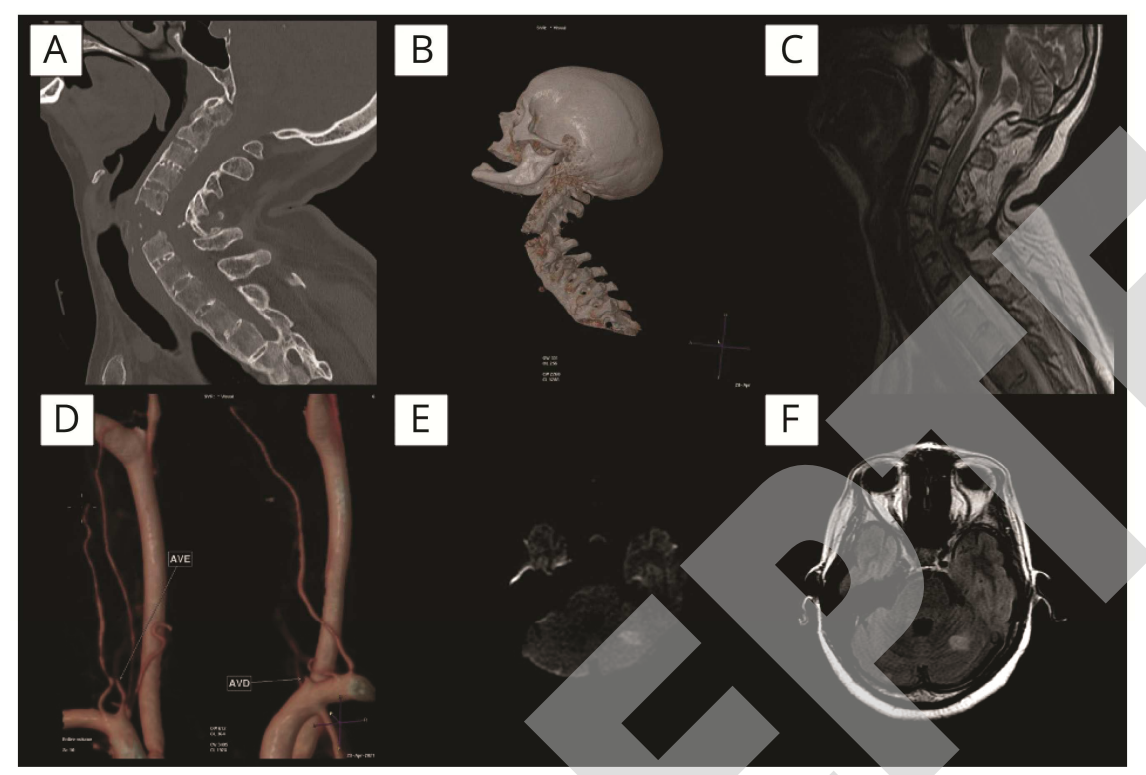




\section{Neurology}

\section{Spinal Cord Injury, Vertebral Artery Dissection, and Cerebellar Strokes After Chiropractic Manipulation}

Gabriel Bortoli Ramos, Rebecca Ranzani Martins, Julia Carvalhinho Carlos de Souza, et al. Neurology published online November 18, 2021

DOI 10.1212/WNL.0000000000013078

\section{This information is current as of November 18, 2021}

\section{Updated Information \&} Services

Subspecialty Collections

Errata

Permissions \& Licensing

Reprints including high resolution figures, can be found at:

http://n.neurology.org/content/early/2021/11/18/WNL.0000000000013078. citation.full

This article, along with others on similar topics, appears in the following collection(s):

CT

http://n.neurology.org/cgi/collection/ct

Infarction

http://n.neurology.org/cgi/collection/infarction

MRI

http://n.neurology.org/cgi/collection/mri

Spinal cord trauma

http://n.neurology.org/cgi/collection/spinal_cord_trauma

Spinal cord trauma; see Trauma/spinal cord trauma

http://n.neurology.org/cgi/collection/spinal_cord_trauma-see_trauma-spinal _cord_trauma

An erratum has been published regarding this article. Please see next page or:

/content/99/1/42.full.pdf

Information about reproducing this article in parts (figures,tables) or in its entirety can be found online at:

http://www.neurology.org/about/about_the_journal\#permissions

Information about ordering reprints can be found online:

http://n.neurology.org/subscribers/advertise

Neurology ${ }^{\circledR}$ is the official journal of the American Academy of Neurology. Published continuously since 1951, it is now a weekly with 48 issues per year. Copyright (C) 2021 American Academy of Neurology. All rights reserved. Print ISSN: 0028-3878. Online ISSN: 1526-632X.

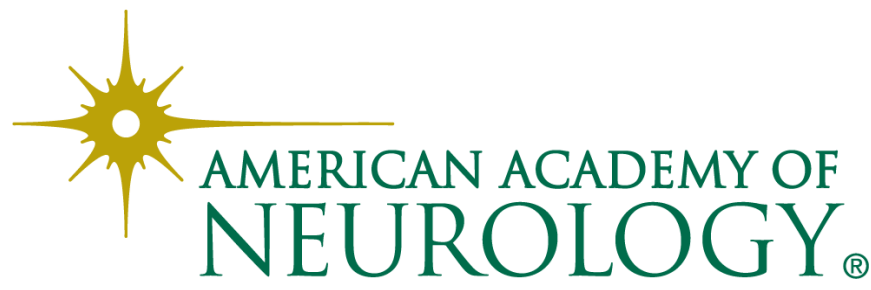




\section{Disputes \& Debates: Editors' Choice}

Steven Galetta, MD, FAAN, Editor

Aravind Ganesh, MD, DPhil, FRCPC, Deputy Editor

Ariane Lewis, MD, Deputy Editor

James E. Siegler III, MD, Deputy Editor

\section{Editors' Note: Shoulder-Tap Test for Functional Gait Disorders: A Sign of Abnormal Anticipatory Behavior}

Dr. Coebergh and colleagues described exaggerated postural responses in 25 patients with functional gait disorders, following a sudden shoulder tap applied by an examiner from behind the patient. In response, Dr. Geroin et al. comment that in their own population of patients with functional neurologic disorders, a light touch on the shoulders seemed to provide fewer false-positive results, given that a sudden tap could evoke exaggerated responses in patients with disorders such as hyperekplexia or stiff-person syndrome. They also note that it is not necessary to see an intact response to a retropulsion test in patients with functional gait disorders to flag their shoulder-test response as incongruent. Responding to these comments, Dr. Coebergh agrees that a light touch would likely suffice, noting that the actual contact with the shoulders in the described shoulder-tap test is light, while also highlighting work being performed by their group in identifying sensory thresholds of force at which incongruent postural responses occur in such patients. To illustrate the potential value of observing incongruity in responses to shoulder-tap vs retropulsive stimuli in patients with functional gait disorder, Dr. Coebergh highlights a case of a patient with stiff-person syndrome misdiagnosed as a functional neurologic disorder, in whom such incongruity was not observed. This exchange demonstrates the enduring value of systematic clinical examinations and observations in improving the diagnosis of functional neurologic disorders.

\section{Reader Response: Shoulder-Tap Test for Functional Gait Disorders: A Sign of Abnormal Anticipatory Behavior}

\footnotetext{
Christian Geroin (Verona, Italy), Jorik Nonnekes (Nijmegen, the Netherlands), Serena Camozzi (Verona, Italy), Bastiaan R. Bloem (Nijmegen, the Netherlands), and Michele Tinazzi (Verona, Italy) Neurology ${ }^{\circledR}$ 2022;99:38-39. doi:10.1212/WNL.0000000000200866
}

In a recent report, Coebergh et al. ${ }^{1}$ introduced the shoulder-tap test, which helps to reveal incongruity in patients with functional gait disorders. Specifically, the authors observed an exaggerated postural response in $82 \%$ of patients, following a gentle shoulder tap applied from behind the patient. ${ }^{1}$ We applaud the described approach because incongruencies of symptoms and signs have great diagnostic value when diagnosing a functional neurologic disorder. ${ }^{2-4} \mathrm{We}$ hope to extend the findings of Coebergh et al. by making 2 additional points.

As observed in our population of functional neurologic disorders, a light touch on the shoulders instead of a shoulder tap might be more informative and provide fewer false-positive results because a sudden tap can evoke an exaggerated postural response in patients with hyperekplexia or stiff-person syndrome. This movement would then incorrectly be classified as an incongruent response. In addition, the authors state that incongruity involves an abnormal response to the shoulder tap combined with an intact response to the normal retropulsion test. We would argue 
that an isolated abnormal response to a mere touch to the shoulders would already suggest incongruity, with a consideration for rare organic disorders such as hyperekplexia and stiff-person syndrome.

1. Coebergh J, Zimianiti I, Kaski D. Shoulder-tap test for functional gait disorders: a sign of abnormal anticipatory behavior. Neurology. 2021;97(23):1070-1071.

2. Espay AJ, Aybek S, Carson A, et al. Current concepts in diagnosis and treatment of functional neurological disorders. JAMA Neurol. 2018;75(9):1132-1141.

3. Nonnekes J, Růžička E, Serranová T, Reich SG, Bloem BR, Hallett M. Functional gait disorders: a sign-based approach. Neurology. 2020; 94(24):1093-1099.

4. Gupta A, Lang AE. Psychogenic movement disorders. Curr Opin Neurol. 2009;22(4):430-436.

Copyright (c) 2022 American Academy of Neurology

\section{Author Response: Shoulder-Tap Test for Functional Gait Disorders: A Sign of Abnormal Anticipatory Behavior}

Jan A. Coebergh (Chertsey, UK)

Neurology ${ }^{\circledR}$ 2022;99:39. doi:10.1212/WNL.0000000000200867

We welcome the comments by Dr. Geroin et al. ${ }^{1}$ on our article. We agree that a light touch may be a sufficient stimulus to induce an anticipatory postural response in patients with functional gait disorders, without the need for a "tap." Although the video linked to our article shows a rapid downward movement of the examiner's hands onto the shoulders, the actual contact with the shoulders is in fact light, lending credence to our esteemed colleagues' observations. Whether there exists a sensory threshold of force, at which an incongruent or inappropriate postural response occurs following a touch to the shoulders, is an interesting question that we hope to be able to address in our future work. In fact, we are extending our work to explore the shoulder-tap test in elderly people with a fear of falling-a common symptom in which abnormal anticipatory behaviors likely exist and identification of such thresholds may be of particular interest.

We also agree that an abnormal response to a light shoulder tap would be an incongruent response to such a stimulus, but, as we describe in our article, a normal response to a retropulsive stimulus or a pull after an abnormal shoulder tap provides further evidence of incongruity.

Finally, we have recently seen a patient with stiff-person syndrome who had been previously misdiagnosed as having a functional neurologic disorder; the clue to the actual diagnosis, beyond the presence of auditory startle and hyperlordosis, was indeed the absence of any incongruity in postural responses. We therefore appreciate the comments regarding this rare but treatable disorder and the importance of avoiding undue force during a shoulder tap or touch that would render interpretation of the ensuing behavior more challenging.

1. Coebergh J, Zimianiti I, Kaski D. Shoulder-tap test for functional gait disorders: a sign of abnormal anticipatory behavior. Neurology. 2021;97(23):1070-1071

Copyright @ 2022 American Academy of Neurology 
CORRECTION

\section{In Vivo Diagnosis of Synucleinopathies}

A Comparative Study of Skin Biopsy and RT-QuIC

Neurology ${ }^{\circledR}$ 2022;99:40-42. doi:10.1212/WNL.0000000000200689

In the article "In Vivo Diagnosis of Synucleinopathies: A Comparative Study of Skin Biopsy and RT-

QuIC" by Donadio et al., the first paragraph of the Methods section should indicate that of the 31

Table 4 Immunofluorescence and Real-Time Quaking-Induced Conversion (RT-QulC) Results of Skin and CSF Samples

\begin{tabular}{|c|c|c|c|c|c|c|}
\hline \multirow[b]{2}{*}{ Variable } & \multirow[b]{2}{*}{ Cases, $\mathbf{n}$} & \multirow[b]{2}{*}{ Diagnosis } & \multicolumn{3}{|l|}{ Skin } & \multirow[b]{2}{*}{ CSF, RT-QuIC } \\
\hline & & & Protocol & Immunofluorescence & RT-QuIC & \\
\hline \multirow[t]{19}{*}{ Synucleinopathies } & 1 & PD (1 patient $\left.{ }^{\mathrm{a}}\right)$ & III & + & + & + \\
\hline & 2 & PD (6 patients $\left.{ }^{a}\right)$ & III & + & + & ND \\
\hline & 3 & PD (1 patient) & II & + & + & + \\
\hline & 4 & PD (3 patients $\left.{ }^{a}\right)$ & II & + & + & ND \\
\hline & 5 & PD (5 patients) & III & + & + & ND \\
\hline & 6 & PD (1 patient $\left.{ }^{\mathrm{a}}\right)$ & 1 & - & + & ND \\
\hline & 7 & MSA-C (1 patient) & III & + & + & + \\
\hline & 8 & MSA-P (3 patients) & 1 & + & + & ND \\
\hline & 9 & MSA-P (1 patient) & 1 & - & - & ND \\
\hline & 10 & MSA-P (1 patient) & 1 & + & - & ND \\
\hline & 11 & MSA-P (1 patient) & 1 & - & + & ND \\
\hline & 12 & MSA-C (1 patient) & 1 & + & ND & - \\
\hline & 13 & Lewy body dementia (1 patient) & III & + & - & ND \\
\hline & 14 & Lewy body dementia (2 patients) & ॥ & + & + & + \\
\hline & 15 & Lewy body dementia (1 patient) & 1 & + & + & + \\
\hline & 16 & Lewy body dementia (1 patient) & 1 & + & + & ND \\
\hline & 17 & PAF (1 patient) & II & + & + & + \\
\hline & 18 & PAF (1 patient) & $\|$ & - & - & - \\
\hline & 19 & PAF (1 patient) & 1 & + & + & ND \\
\hline \multirow[t]{10}{*}{ Nonsynucleinopathies } & 1 & AD (5 patients) & III & - & - & - \\
\hline & 2 & AD (2 patients) & III & - & + & - \\
\hline & 3 & AD (1 patient) & III & - & - & ND \\
\hline & 4 & AD (1 patient) & III & -- & + & ND \\
\hline & 5 & AD (2 patients) & ॥ & - & - & - \\
\hline & 6 & AD (1 patient) & II & - & - & ND \\
\hline & 7 & AD (2 patients) & 1 & - & - & - \\
\hline & 8 & AD (1 patient) & 1 & - & - & ND \\
\hline & 9 & PSP (1 patient) & III & - & + & ND \\
\hline & 10 & PSP (2 patients) & II & - & - & - \\
\hline
\end{tabular}


Table 4 Immunofluorescence and Real-Time Quaking-Induced Conversion (RT-QuIC) Results of Skin and CSF Samples (continued)

\begin{tabular}{|c|c|c|c|c|c|c|}
\hline \multirow[b]{2}{*}{ Variable } & \multirow[b]{2}{*}{ Cases, $\mathbf{n}$} & \multirow[b]{2}{*}{ Diagnosis } & \multicolumn{3}{|l|}{ Skin } & \multirow[b]{2}{*}{ CSF, RT-QuIC } \\
\hline & & & Protocol & Immunofluorescence & RT-QuIC & \\
\hline & 11 & CBS (1 patient) & II & - & - & ND \\
\hline & 12 & CBS (1 patient) & ॥ & - & + & ND \\
\hline & 13 & Vascular parkinsonism (3 patients ${ }^{\mathrm{a}}$ ) & III & - & - & ND \\
\hline & 13 & Vascular parkinsonism (1 patient) & II & - & - & - \\
\hline & 14 & Vascular parkinsonism (2 patients ${ }^{a}$ ) & II & - & - & ND \\
\hline & 15 & Vascular parkinsonism (2 patients) & I & - & - & - \\
\hline & 16 & latrogenic parkinsonism (1 patient) & I & - & - & ND \\
\hline & 17 & Autoimmune parkinsonism (1 patient) & II & - & - & - \\
\hline & 18 & Vascular dementia (1 patient) & III & - & - & - \\
\hline & 19 & Vascular dementia (1 patient) & II & - & + & - \\
\hline & 20 & ALS (1 patient) & III & - & + & - \\
\hline & 21 & ALS (1 patient) & II & - & + & - \\
\hline & 22 & ALS (3 patients) & I & - & - & - \\
\hline & 23 & ALS (1 patient) & I & - & + & ND \\
\hline \multirow[t]{19}{*}{ Controls } & 1 & Small fiber neuropathy (3 patients) & III & - & - & - \\
\hline & 2 & Small fiber neuropathy ( 2 patients) & III & - & - & ND \\
\hline & 3 & Small fiber neuropathy (1 patient) & I & - & - & - \\
\hline & 4 & Small fiber neuropathy ( 1 patient) & I & - & - & ND \\
\hline & 5 & Peripheral neuropathy (1 patient) & III & - & + & ND \\
\hline & 6 & Peripheral neuropathy (1 patient) & II & - & - & - \\
\hline & 7 & Peripheral neuropathy (1 patient) & II & - & + & - \\
\hline & 8 & Peripheral neuropathy (1 patient) & 1 & - & - & - \\
\hline & 9 & Peripheral neuropathy (1 patient) & I & - & - & ND \\
\hline & 10 & CIDP (1 patient) & I & - & - & - \\
\hline & 11 & Wernicke encephalopathy (1 patient) & I & - & - & - \\
\hline & 12 & Depression (1 patient) & III & - & - & - \\
\hline & 13 & Depression (2 patients) & III & - & - & ND \\
\hline & 14 & Depression (2 patients) & II & - & - & - \\
\hline & 15 & Cerebral vasculitis (1 patient) & II & - & - & - \\
\hline & 16 & Cerebral vasculitis (1 patient) & II & - & ND & - \\
\hline & 17 & SCD (1 patient) & III & - & + & - \\
\hline & 18 & SCD (1 patient) & II & - & - & - \\
\hline & 19 & Stiff-person syndrome (1 patient) & I & - & - & ND \\
\hline
\end{tabular}

Abbreviations: $A D=$ Alzheimer disease; $A L S=$ amyotrophic lateral sclerosis; $C B S=$ corticobasal syndrome; CIDP = chronic inflammatory demyelinating polyneuropathy; MSA-C = multiple system atrophy, cerebellar type; MSA-P = multiple system atrophy, parkinsonian type; ND = not done; PAF = pure autonomic failure; PD = Parkinson disease; PSP = progressive supranuclear palsy; SCD = subjective cognitive disorder.

a Patients from whom 10 skin samples were taken; in the remaining patients, 8 skin samples were taken combining the routine and study protocols (see Methods for more details). 
patients with synucleinopathies, 15 were patients with Parkinson disease, and of the 38 patients with nonsynucleinopathies, 7 were patients with vascular parkinsonism. The authors regret the errors.

In addition, the Diagnosis column of Table 4 should read "patients" in parentheses, not "points." See the corrected Table 4. The publisher regrets the errors.

\section{Reference}

1 Donadio V, Wang Z, Incensi A, et al. In vivo diagnosis of synucleinopathies: a comparative study of skin biopsy and RT-QuIC. Neurology. 2021;96(20): e2513-e2524.

\section{NOTICE}

\section{Dual Publication: Spinal Cord Injury, Vertebral Artery Dissection, and Cerebellar Strokes After Chiropractic Manipulation}

Neurology ${ }^{\circledR}$ 2022;99:42. doi:10.1212/WNL.0000000000200334

The Editors of Neurology ${ }^{\circledR}$ and Internal and Emergency Medicine issue a notice of publication of 2 reports of a single patient written by 2 different author groups.

Macêdo MB, Shinjo SK, Domiciano DS. Breaking the Diagnosis: Ankylosing Spondylitis Evidenced by Cervical Fracture following Spine Manipulation. Intern Emerg Med 2021. DOI: $10.1007 / \mathrm{s} 11739-021-02829-2$.

Ramos GB, Martins RR, Souza JCC, Falcão FCSEB, Lopes CCB, Andrade ALG, Silva GD. Spinal Cord Injury, Vertebral Artery Dissection, and Cerebellar Strokes after Chiropractic Manipulation. Neurology 2021. DOI: 10.1212/WNL.0000000000013078.

The first article was published in Internal and Emergency Medicine. The journal received it on July 20, 2021, accepted it on August 10, 2021, and published it online on August 20, 2021. The second article was published in Neurology. It was received on July 16, 2021, accepted on November 4, 2021, and published online on November 18, 2021. The 2 case reports were based on the same patient and shared a common image.

Both case reports were written by authors from the Faculdade de Medicina, Universidade de São Paulo. The authors of the article published in Internal and Emergency Medicine were affiliated with the Department of Rheumatology, and the authors of the Neurology article were affiliated with the Department of Neurology.

The authors of both articles were contacted and asked for an explanation for the dual publication. Both teams of authors explained that they cared for the patient during the hospital admission and that they were unaware of the submission by the other team. The focus of the articles is different: one focused on bone injury and emergency care and the other on the neurologic aspects of the case. Both author groups apologize for the duplicate submissions.

A reader first notified Neurology that the case had been published in the 2 journals and a brief report was submitted to Neurology's Disputes \& Debates section on November 29, 2021. ${ }^{1}$

\section{Reference}

1. Trager RJ, Garcia JA. Disputes \& Debates: Rapid Online Correspondence. Reader Response: Spinal Cord Injury, Vertebral Artery Dissection, and Cerebellar Strokes after Chiropractic Manipulation. n.neurology.org/content/reader-response-spinal-cord-injury-vertebral-arterydissection-and-cerebellar-strokes-after.

Copyright (c) 2022 American Academy of Neurology 